\title{
Review
}

\section{The Potential of Amniotic Fluid-Derived Extracellular Vesicles to Treat Severe Acute Respiratory Syndrome Coronavirus 2 Infection Versus Hydroxychloroquine in Human Patients}

\author{
lan A.White, PhD* \\ Chief Scientific Officer, Neobiosis, LLC, I 735 Bay Drive, Miami Beach, FL 33 I I , USA \\ "Corresponding author \\ lan A. White, PhD \\ Chief Scientific Officer, Neobiosis, LLC, 1735 Bay Drive, Miami Beach, FL 33।4I, USA; E-mail:drwhite@neobiosis.com
}

\section{Article information}

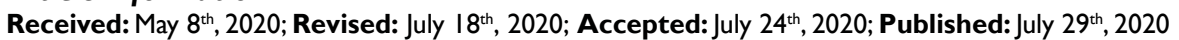

\section{Cite this article}

White IA. The potential of amniotic fluid-derived extracellular vesicles to treat severe acute respiratory syndrome coronavirus 2 infection versus hydroxychloroquine in human patients. Epidemiol Open J. 2020; 5(I): 8-12. doi: I0.17।40/EPOJ-5-II8

\section{ABSTRACT}

Recently a lot of attention has been focused on fast-tracking repurposed drugs for the treatment of a novel coronavirus; severe acute respiratory syndrome coronavirus 2 (SARS-CoV-2), the causative agent leading to the devastating coronavirus disease-2019 (Covid-19). One of the first and most well-known examples is hydroxychloroquine (HCQ), which has been used for years as a treatment for malaria, rheumatoid arthritis and lupus. HCQ was rolled out as a miracle treatment for Covid-19, which was inexpensive, effective and causing few side-effects. However, its effectiveness in treating Covid-19 has recently been questioned leaving doctors and patients confused and desperate. Here the proposed mechanisms of action of HCQ are discussed and compared with an inexpensive, safe and effective alternative, which is derived from natural, healthy amniotic fluid.
\end{abstract}

Keywords

Covid-19; SARS-CoV-2; Amniotic fluid; CytoSomes.

\section{INTRODUCTION}

$\mathrm{T}$ he highly pathogenic severe acute respiratory syndrome coronavirus (SARS-CoV) is a lethal zoonotic virus that has emerged into human populations these past 15 -years. These corona viruses are associated with novel respiratory syndromes that spread from person-to-person via close contact, resulting in high morbidity and mortality caused by the progression to acute respiratory distress syndrome (ARDS). Mortality associated with cytokine storm (immune dysregulation) resulting in multiorgan failure is rising at an alarming rate in the US. This is in part due to the relatively high R0 (contagion ratio) and devastating hypoxia, particularly in the aged population. Hydroxychloroquine (HCQ) an antimalarial drug, can be used in the regulation of the immune system, e.g. it has previously been used in the treatment of autoimmune diseases. The immunosuppressive effects of HCQ have also been beneficial to patients with severe inflammatory complications from several viral diseases ${ }^{1}$ and for this reason HCQ has received a lot of attention during this current SARS-CoV-2 pandemic.
It is abundantly clear that immune modulation is required to counteract the cytokine storm and tissue injury observed in severe and fatal SARS-CoV-2 cases. HCQ certainly has demonstrable anti-inflammatory effects in multiple viral infections, including SARS-CoV-1, however, immune suppression is not desirable in the case of severe SARS-CoV-2 patients. Rather, immune modulation, which the virus actively subverts, is critical for acute recovery and avoidance of long-term pathology due to pulmonary scarring. To this end we propose the use of extracellular vesicles (EVs) derived from full-term, healthy amniotic fluid (AF). This fluid is collected by AABB-accredited and Food and Drug Administration (FDA)approved cord blood banks with maternal consent and following extensive infectious disease testing, which is standard for all transplantable human tissue, including blood donations. Amniotic EVs, which we call "CytoSomes", have been used clinically with demonstrable effects on immune modulation, infection and tissue repair. Indications ranging from autoimmune disease to neurodegenerative disease have been treated with CytoSomes. While the clinical application of this product is relatively new, research into 
the biological effects of amniotic fluid and amniotic fluid stem cellderived EVs has been ongoing for decades. ${ }^{2-8}$ Relevant to SARSCoV-2 infection, these EVs are documented to deliver potent antimicrobial effects, are highly immunomodulatory and stimulate tissue growth and repair. These foci are critical in the treatment of severe hypoxia and organ failure due to SARS-Cov-2 infection.

\section{HYDROXYCHLOROQUINE VERSUS AMNIOTIC EVS FOR THE TREATMENT OF SARS-CoV-2}

Diseases in humans are typically characterized by a polarization of a pro-inflammatory environment orchestrated by Type $1 \mathrm{~T}$-cells and classically activated macrophages (M1) and a wound healing, anti-inflammatory environment driven by alternatively activated macrophages (M2). ${ }^{9}$ M1 macrophagesarepro-inflammatory and polarize in response to viral infection, however over stimulation results in tissue injury. M1 macrophages produce nitricoxide (NO) or reactive oxygen intermediates (ROI) to protect against bacteria and viruses, which is mediated by interleukin-1 (IL-1) and tumor necrosis factor-alpha (TNF $\alpha)$, primarily. ${ }^{10}$ Although IL-1 signaling plays pivotal roles in immunity, sterile inflammation and metabolism, excessive overproduction or dysregulation of IL-1 is highly detrimental and contributes to auto-inflammatory diseases, autoimmune encephalomyelitis, cytokine storm and tissue damage, including pulmonary injury such as SARS asseen with SARS-CoV. IL-1 production is therefore extensively regulated and the margin between clinical benefit and undesirable pathogenic effects for IL-1 is exceedingly narrow. A balance is required between the polarization of M1 macrophages and M2 macrophages, which are anti-inflammatory and contribute to wound healing.

Inflammation is the physiological response to a variety of injuries, which leads to a complex immune response. In the case of viral infections this is characterized by immune cell recruitment and release of molecular mediators acting towards pathogen elimination, which is mediated by M1 macrophages. This is then followed by an exquisitely orchestrated process directed towards repairing damaged tissue and restoring homeostasis, mediated by a switch from M1 to M2 macrophages. HCQ has received much attention recently as a potential treatment for SARS-CoV-2.

Hydroxychloroquine is generally well tolerated and exhibits an anti-inflammatory profile because it down-regulates the effects of M1 macrophages. The main factory for generating viral particles is within human bronchial epithelial cells, alveolar epithelial cells and the endosomes of human alveolar macrophages, which are the primary targets of SARS-CoV-2 in humans. ${ }^{11,12}$ Generally, it is accepted that directly after virus recognition, macrophages endocytose the invading pathogens, polarize to the M1 phenotype and present viral peptides via the major histocompatibility complex (MHC) to T-lymphocytes that will then produce IFN- $\gamma$ and other molecules to recruit effect or immune cells and induce an inflammatory response capable of controlling viral replication. ${ }^{12}$ Unfortunately, this pro-inflammatory state provides the stimuli to drive infected macrophages from the periphery deep into tissues, which promotes viral dissemination during the early infection stage. Such a feed-back loop results in extensive viral replication, tissue injury and an inhibition of M2-mediated tissue repair. In weaker patients, this cycle ultimately results in severe pulmonary njury and death. One of the main mechanisms of action of HCQ, other than the increasing the $\mathrm{pH}$ inside macrophage endosomes, which alters the processing of peptide antigens, ${ }^{13}$ and its role as an ionophore for zinc uptake into infected cells, is to kill off the M1 macrophages that are contributing to inflammation and viral replication. ${ }^{10} \mathrm{HCQ}$ is highly cytotoxic to macrophages. However, by simply eliminating M1 macrophages, as a means to suppress inflammation and viral replication, neglects the requirement to repair tissue injury sustained by viral proliferation. The consequences of this can be varied, but will likely include acute fatalities due to inadequate alveolar repair and pathological pulmonary scarring, which will have negative long-term clinical outcomes.

While HCQ has demonstrated positive results for some patients, a growing number of patients are not responding to standard or escalating doses of HCQ. While the data is not yet available, and likely not being collected, it is highly probable that HCQ is in effective for the large-scale treatment of SARS-CoV-2, due to macrophage cytotoxicity and subsequent suboptimal M2-mediated pulmonary tissue repair. Macrophage polarization is connected to the course of resolving inflammation, where tissues are typically repaired after infection, however, they also play a role in "non-resolving inflammation", where a pathogen can prolong inflammation. Such polarization might occur at any time during the inflammatory process, and M1/M2 macrophages have different functions that are required to destroy pathogens or repair the inflammation and maintain homeostasis. Elimination of these mediators is akin to knocking the building down to put out the fire. A more thoughtful approach would be to eliminate the polarization of M1 macrophages and promote the polarization of M2 macrophages. A strategy that combines viral replication inhibitors, such as vitamin $C$ and the peptide Thymosin alpha-1 (NK activator), elimination of M1-mediated pro-inflammatory cytokine storm and promotion of M2 pulmonary tissue would likely have positive acute outcomes on survival and long-term effects on pulmonary fibrosis (particularly for those who have been ventilated). Supplementation with the antioxidant L-NAC/glutathione (cardiac and pulmonary nerve support) and pro-reparative peptides such as body protein compound-157 (BPC-157) may complement such treatments.

\section{WHY AMNIOTIC FLUID-DERIVED EXTRACELLULAR VESICLES? |}

News reports from the initial wave of Covid-19 described a terminal patient in an intensive care unit (ICU) in Italy was treated with a combination approach that included the use of "stem cells". The patient subsequently improved and was discharged from hospital. This treatment resulted in a lot of discussion regarding the use of stem cells to treat SARS-CoV-2 patients. It is agreed among scientists that stem cell therapy, or more accurately cytotherapy, does not result in tissue regeneration by direct differentiation of the cells, but rather repair is mediated by the cells through the liberation of EVs, which are how the cells communicate to injured tissue. In nature, these extracellular signaling vesicles accumulate in body fluids such as blood, breast milk, urine and amniotic fluid. In 
experiments where amniotic fluid "stem cells" are used to promote tissue repair, the effects are driven by the EVs that they produce, which instruct the tissue how to repair and provide the building blocks with which to do so. We have developed proprietary methods of isolating EVs from amniotic fluid (cytosomes), which are derived from amniotic stem cells, regenerative epithelial cells and monocytes, among others. These EVs are potent mediators of tissue repair and immune modulation.

For over a decade, scientists at Harvard University have been studying a process called hetero chronic parabiosis ("hetero"-different, "chronic"-age, "para"- together, "biosis"-living) in mice. In their experiments they connected the blood supply of an old mouse together with that of a young mouse. Over time scientists recorded changes in both individuals. The young mouse started demonstrating signs of accelerated aging, while the old mouse showed signs of improved tissue repair, immune modulation and anti-aging. It was later discovered that it was not necessary to connect the blood supply to observe this phenomenon. By simply harvesting the blood plasma from a young mouse and administering it, intravenous, to an old mouse, the old mouse began to exhibit the same anti-aging effects and improved tissue regeneration.

We have known for some time that young individuals have enhanced tissue regenerative and immune modulation capacity compared to aged individuals, ${ }^{15}$ and it is likely that this has a direct contribution to why SARS-CoV-2 is devastating for the elderly, while relatively benign in the young. The young are able to rapidly repair injury to the lungs caused by the virus and robustly control the inflammation that leads to cytokine storm. Interestingly, a fetus in utero has a $100 \%$ capacity to repair tissue if injured experimentally. This regenerative capacity declines precipitously following birth and continues to drop as we age (Figure 1). ${ }^{15-17}$ Indeed, these are the mechanism by which all species age and eventually die.
A natural example of heterochronic parabiosis exists, which we call pregnancy. During pregnancy a young individual shares the blood supply with an older individual, the mother. The signals that are exchanged between mother a child are packaged into extracellular vesicles and these EVs accumulate in the amniotic fluid (AF). The fetus drinks the amniotic fluid during pregnancy and it has been demonstrated that the EVs within AF are critical for gut development. If there is insufficient AF present during pregnancy fetal gastrointestinal development is severely compromised. ${ }^{18,19}$ Indeed, fetal exposure to AF is critical for robust wound healing $^{20}$ and as AF enters the fetal lungs it is essential for lung development and function. ${ }^{21-25}$ Moreover, the effects of EVs generated during pregnancy are not limited to the fetus, but also have positive effects on the mother. The stresses and rigors of pregnancy would be fatal without physiological changes to the mother. During the final trimester the mother experiences a cardiac output equivalent to that of an Olympic athlete. Cardiac output triples and the lungs must respond to the increased demand for oxygen. ${ }^{26,27}$ Communication between the fetus and the mother is mediated by amniotic EVs, which results in the effects seen in experimental heterochonic parabiosis. In the case of natural heterochonic parabiosis maternal cardiac and pulmonary output increases, the material immune system is modulated, as to not reject the fetus, and even some cancers are inhibited. ${ }^{28}$ Cardiac and pulmonary support and immune modulation are critical for aging patients who are afflicted with SARS-CoV-2. Their innate tissue regenerative capacity and ability to modulate a cytokine storm, as seen with this virus, is severely compromised. Amniotic fluid EVs have been studied in recent years for their potent capacity to confer regenerative potential and immune modulation to recipient patients. ${ }^{2-8,29,30} \mathrm{We}$ therefore propose that IV infusion of amniotic fluid EVs (Cytosomes) isolated from healthy, full-term pregnancies may help terminal SARS-CoV-2 patients modulate the inflammation and tissue damage, which would otherwise result in organ failure and death. Furthermore, in a cruel twist for patients recovered with hydroxy-

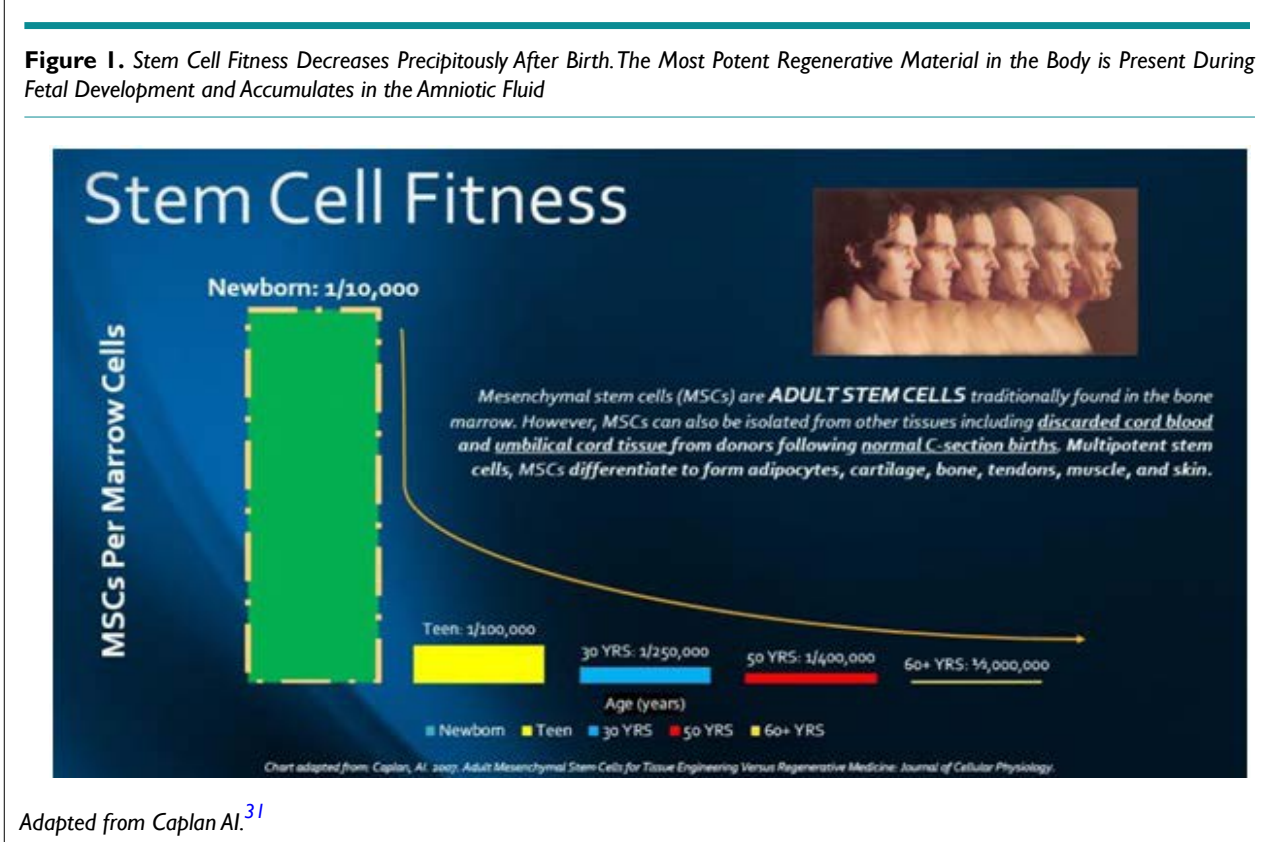


chloroquine, the lack of M2 macrophages and subsequent reduction in tissue repair will likely result in substantial pulmonary fibrosis, which will have ongoing, long-term health issues. Injection of amniotic fluid stem cells, which work through the release of EVs, has been demonstrated to delay the progression of renal fibrosis. Treated animals exhibited decreased recruitment and activation of M1-type macrophages and a higher proportion of M2-type macrophages, which promote tissue regeneration. ${ }^{29}$ A functional M2 pro-regenerative program is critical for SARS-CoV-2 patients to prevent long-term scarring and chronic illness.

\section{CONCLUSION}

SARS-CoV-2 is a devastating viral infection that results in hypoxia, organ failure and death primarily in older patients. Hydroxychloroquine has emerged as a leading contender in the pursuit of treatments for severe and terminal cases. This antimalarial drug works by affecting the macrophages, which are among the cells targeted by this corona virus. The key to the function of this drug is that it 1) reduces macrophage presentation of viral particles, which can reduce the amplitude of an immune response, 2) acts as an ionophore to drive zinc into infected cells, and 3) kills the macrophages which proliferate in response to viral infection and are hijacked by the virus in order to replicate. The issue is that for an effective recovery $\mathrm{M} 1$, pro-inflammatory, macrophages need to polarize to M2, regenerative, macrophages to facilitate repair to the damaged lung tissue. Unfortunately, elimination of macrophages will not be effective for all patients as bronchial epithelial cells and alveolar epithelial cells are also hijacked for viral replication and other antigen presenting cells can promote inflammation. Moreover, suppression of M2 polarization can have substantial negative outcomes for pulmonary fibrosis (scarring) following recovery from this disease and the injury induced by ventilation. As an alternative, we have documented evidence of the effects of amniotic fluid extracellular vesicles (cytosomes) on inflammation and tissue repair in adult recipients. SARS-CoV-2 primarily affects older patients. Older patients are severely compromised in their innate ability to repair tissue and modulate inflammation. By transplanting EVs from very young sources (amniotic fluid) into adult SARS-CoV-2 patients, we believe we can supplement the lack of tissue repair and facilitate recovery by a natural process, which does not result in immune suppression, but rather favors immune modulation and pulmonary regeneration.

\section{REFERENCES}

1. Totura AL, Bavari S. Broad-spectrum coronavirus antiviral drug discovery. Expert Opin Drug Discov. 2019; 14: 397-412. doi: 10.1080/17460441.2019.1581171

2. Antounians L, Tzanetakis A, Pellerito O, Catania VD, Sulistyo A, Montalva L, et al. The regenerative potential of amniotic fluid stem cell extracellular vesicles: Lessons learned by comparing different isolation techniques. Sci Rep. 2019; 9: 1837. doi: 10.1038/ s41598-018-38320-w

3. Balbi C, Lodder K, Costa A, Moimas S, Moccia F, van Herwaarden $\mathrm{T}$, et al. Supporting data on in vitro cardioprotective and proliferative paracrine effects by the human amniotic fluid stem cell secretome. Data Brief. 2019; 25: 104324. doi: 10.1016/j. dib.2019.104324

4. Balbi C, Piccoli M, Barile L, Papait A, Armirotti A, Principi E, et al. First characterization of human amniotic fluid stem cell extracellular vesicles as a powerful paracrine tool endowed with regenerative potential. Stem Cells Transl Med. 2017; 6: 1340-1355. doi: 10.1002/sctm.16-0297

5. Beretti F, Zavatti M, Casciaro F, Comitini G, Franchi F, Barbieri $\mathrm{V}$, et al. Amniotic fluid stem cell exosomes: Therapeutic perspective. Biofactors. 2018; 44: 158-167. doi: 10.1002/biof.1407

6. Loukogeorgakis SP, De Coppi P. Stem cells from amniotic fluid-Potential for regenerative medicine. Best Pract Res Clin Obstet Gynaecol. 2016; 31: 45-57. doi: 10.1016/j.bpobgyn.2015.08.009

7. Mirabella T, Poggi A, Scaranari M, Mogni M, Lituania M, Baldo C, et al. Recruitment of host's progenitor cells to sites of human amniotic fluid stem cells implantation. Biomaterials. 2011; 32: 42184227. doi: 10.1016/j.biomaterials.2010.12.028

8. Zhao B, Zhang Y, Han S, Zhang W, Zhou Q, Guan H, et al. Exosomes derived from human amniotic epithelial cells accelerate wound healing and inhibit scar formation. J Mol Histol. 2017; 48: 121-132. doi: 10.1007/s10735-017-9711-x

9. Atri C, Guerfali FZ, Laouini D. Role of human macrophage polarization in inflammation during infectious diseases. Int J Mol Sci. 2018; 19(6): 1801. doi: 10.3390/ijms19061801

10. Perecko T, Kassab RB, Vasicek O, Pekarova M, Jancinova V, Lojek A. The effects of chloroquine and hydroxychloroquine on nitric oxide production in RAW 264.7 and bone marrow-derived macrophages. Folia Biol (Praha). 2014; 60 Suppl 1: 39-44.

11. Hartwig SM, Holman KM, Varga SM. Depletion of alveolar macrophages ameliorates virus-induced disease following a pulmonary coronavirus infection. PLoS One. 2014; 9: e90720. doi: 10.1371/journal.pone.0090720

12. Miura TA and Holmes KV. Host-pathogen interactions during coronavirus infection of primary alveolar epithelial cells. J Lenkoc Biol. 2009; 86: 1145-1151. doi: 10.1189/jlb.0209078

13. Al-Bari MAA. Targeting endosomal acidification by chloroquine analogs as a promising strategy for the treatment of emerging viral diseases. Pharmacol Res Perspect. 2017; 5: e00293. doi: $10.1002 / \operatorname{prp} 2.293$

14. Loffredo FS, Steinhauser ML, Jay SM, Gannon J, Pancoast JR, Yalamanchi P, et al. Growth differentiation factor 11 is a circulating factor that reverses age-related cardiac hypertrophy. Cell. 2013; 153: 828-839. doi: 10.1016/j.cell.2013.04.015

15. Caplan AI. Adult mesenchymal stem cells for tissue engineering versus regenerative medicine. J Cell Physiol. 2007; 213: 341-347. 
doi: $10.1002 /$ jcp. 21200

16. Porrello ER, Mahmoud AI, Simpson E, Hill JA, Richardson JA, Olson EN, Sadek HA. Transient regenerative potential of the neonatal mouse heart. Science. 2011; 331: 1078-1080. doi: 10.1126/ science. 1200708

17. White IA, Gordon J, Balkan W, Hare JM. Sympathetic reinnervation is required for mammalian cardiac regeneration. Circ Res 2015; 117: 990-994. doi: 10.1161/CIRCRESAHA.115.307465

18. Mulvihill SJ, Stone MM, Fonkalsrud EW, Debas HT. Trophic effect of amniotic fluid on fetal gastrointestinal development. J Surg Res. 1986; 40: 291-296. doi: 10.1016/0022-4804(86)90189-7

19. Trahair JF, Sangild PT. Fetal organ growth in response to oesophageal infusion of amniotic fluid, colostrum, milk or gastrinreleasing peptide: A study in fetal sheep. Reprod Fertil Dev. 2000; 12: 87-95. doi: 10.1071/rd00024

20. Gao X, Devoe LD, Given KS. Effects of amniotic fluid on proteases: A possible role of amniotic fluid in fetal wound healing. Ann Plast Surg. 1994; 33: 128-134; discussion 134-135.

21. Docimo SG, Luetic T, Crone RK, Davies P, Reid L, Retik AB, Mandell J. Pulmonary development in the fetal lamb with severe bladder outlet obstruction and oligohydramnios: A morphometric study. J Urol. 1989; 142: 657-660; discussion 667-668. doi: 10.1016/ s0022-5347(17)38845-6

22. King JC, Mitzner W, Butterfield AB, Queenan JT. Effect of induced oligohydramnios on fetal lung development. Am J Obstet Gynecol. 1986; 154: 823-830. doi: 10.1016/0002-9378(86)90465-5

23. Moessinger AC, Collins MH, Blanc WA, Rey HR, James LS. Oligohydramnios-induced lung hypoplasia: the influence of timing and duration in gestation. Pediatr Res. 1986; 20: 951-954. doi:

\subsection{3/00006450-198610000-00012}

24. Moessinger AC, Singh M, Donnelly DF, Haddad GG, Collins $\mathrm{MH}$, James LS. The effect of prolonged oligohydramnios on fetal lung development, maturation and ventilatory patterns in the newborn guinea pig. J Dev Physiol. 1987; 9: 419-427.

25. Thomas IT, Smith DW. Oligohydramnios, cause of the nonrenal features of Potter's syndrome, including pulmonary hypoplasia. J Pediatr. 1974; 84: 811-815. doi: 10.1016/s0022-3476(74)80753-5

26. Goldschmidt-Clermont DD, White IA. Amniotic Exosomes and Pregnancy. Web site. https://stmsciencemag.org/content/11/492/eaav8521/tab-e-letters. 2019.

27. van Oppen AC, Stigter RH, Bruinse HW. Cardiac output in normal pregnancy: A critical review. Obstet Gynecol. 1996; 87: 310 318. doi: 10.1016/0029-7844(95)00348-7

28. Russo IH, Russo J. Pregnancy-induced changes in breast cancer risk. J Mammary Gland Biol Neoplasia. 2011; 16: 221-233. doi: 10.1007/s10911-011-9228-y

29. Sedrakyan S, Da Sacco S, Milanesi A, Shiri L, Petrosyan A, Varimezova R, et al. Injection of amniotic fluid stem cells delays progression of renal fibrosis. J Am Soc Nephrol. 2012; 23: 661-673. doi: 10.1681/ASN.2011030243

30. Wiklander OPB, Brennan MA, Lotvall J, Breakefield XO, El Andaloussi S. Advances in therapeutic applications of extracellular vesicles. Sci Transl Med. 2019; 11(492): eaav8521. doi: 10.1126/ scitranslmed.aav8521

31. Caplan AI. Adult mesenchymal stemcells for tissue engineeringversus regenerative medicine. Journal of Cellular Physiology. 2007; 341-347. doi: $10.1002 /$ jcp. 21200 